The authors conclude that the loss-of-function mutations seen in Lafora disease disrupt the mechanism by which laforin and malin together regulate MGS and PTG, leading to the accumulation of intracellular glycogen.

Original article Vilchez D et al. (2007) Mechanism suppressing glycogen synthesis in neurons and its demise in progressive myoclonus epilepsy. Nat Neurosci 10: 1407-1413

\section{New findings cast doubt on PrPSc as a diagnostic marker for TSE}

Current diagnostic assays for transmissible spongiform encephalopathies (TSEs), such as Creutzfeldt-Jakob disease, rely on the detection of an abnormal, proteinase-K-resistant form of the host glycoprotein $\operatorname{PrP}\left(\mathrm{PrPSc}^{\mathrm{S}}\right)$ in postmortem brain tissue. However, recent research by scientists at the Roslin Institute in Edinburgh, UK, shows that signs of disease and high infectivity can occur in the presence of little or no detectable PrPSc.

The researchers used a mouse model of TSE generated in a previous study. In this model, transgenic mice homozygous for a mutation at amino acid position 101 in the endogenous PrP gene (101LL mice) were inoculated with hamster scrapie or human GerstmannSträussler-Scheinker disease; these mice show clinical signs and vacuolar pathology consistent with TSE, but low levels of PrP deposition in the brain tissue. Inocula prepared from diseased brain was able to transmit TSE to further 101LL mice, and bioassay analysis demonstrated high titers of infectivity (approximately $10^{7}-10^{9} \mathrm{IU} / \mathrm{g}$ ) in the tissue of these mice, despite low levels of PrP demonstrated by immunohistochemistry with anti-PrP antibodies. Semi-quantitative analysis of immunoblots revealed low levels of proteinase-K-resistant PrP, and conformation-dependent immunoassay and immunoprecipitation detected extremely low levels of PrPSc. No other disease-associated forms of PrP were detected that could account for the high titers of infectivity observed.

The authors conclude that the titer of infectivity does not correlate with the level of PrPSc. Current diagnostic methods might, therefore, underestimate the level of infectivity and fail to identify animals with TSE.

Original article Barron RM et al. (2007) High titres of TSE infectivity associated with extremely low levels of $\mathrm{PrPSc}^{\mathrm{Sc}}$ in vivo. J Biol Chem 282: 35878-35886

\section{Minocycline causes notable deterioration in patients with ALS}

Few treatment options are available for the fatal neurodegenerative disorder amyotrophic lateral sclerosis (ALS). An increasing amount of evidence suggests that the FDA-approved antibiotic minocycline might have neuroprotective effects in animal models of various neurodegenerative disorders, and Gordon et al. have now tested this drug in patients with ALS.

In this multicenter, randomized, controlled phase III trial, 412 patients were randomly assigned to two groups. One group was given minocycline in escalating doses of up to $400 \mathrm{mg}$ per day for 9 months, and the other received placebo.

By use of the revised ALS Functional Rating Scale, the researchers showed that patients with ALS in the minocycline group experienced a significantly greater rate of deterioration than those in the placebo group. Patients taking minocycline also showed a faster decline in forced vital capacity measurements and manual muscle testing scores, but the differences observed between the drug group and the control group were not significant. The groups had similar quality-of-life scores, but adverse events were more common in the minocycline group.

The unexpected finding that minocycline is apparently harmful for patients with ALS has important implications for future trials of this drug in patients with other neurological disorders, say the authors. The finding also has ramifications for the way in which potential neuroprotective agents are screened for use in patients with ALS.

Original article Gordon PH et al. (2007) Efficacy of minocycline in patients with amyotrophic lateral sclerosis: a phase III randomised trial. Lancet Neurol 6: 1045-1053

\section{The role of microglia in the mechanism of neuropathic pain}

The part that activated microglia have in the pathogenesis of neuropathic pain is not well understood. The results of a recent study by Zhang et al. have now shown that both CNSresident microglia and microglia derived from bone marrow $(\mathrm{BM})$ are involved in the response to peripheral nerve injury, and suggest that 